\title{
PROTON INJECTION AND RF CAPTURE IN THE NATIONAL SPALLATION NEUTRON SOURCE
}

\author{
A.U. Luccio * , J. Beebe-Wang, D.Maletic, Brookhaven National Laboratory, Upton, NY 1973 \\ F.W. Jones, TRIUMF, 4004 Wesbrook Mall, Vancouver, B.C. Canada
}

\section{Abstract}

The accelerator system for the 1 to $5 \mathrm{MW}$ National Spallation Neutron Source (NSNS) consists of a linac followed by a $1 \mathrm{GeV}$ proton accumulator ring. Since the ring is a very high current machine, the injection and if capture of the protons is deeply affected by transverse and longitudinal space charge effects. Results of numerical simulation of the process are presented together with considerations on methods and results of space charge treatment in high intensity proton storage rings.

\section{INTRODÜCTION}

The injection and RF capture processes in the accumulator ring is being studied with the code Accsim[1]. The simulation is 6-dimensional, with tracking of a number of representative macro particles through the lattice, in the presence of space charge forces and beam to wall interaction.

A $1 \mathrm{GeV} \mathrm{H}^{-}$beam is injected from the linac, stripped to $\mathrm{H}^{+}$and moved to the equilibrium orbit. Accsim calculates the scattering in the stripping foil, that is traversed a few times by the beam during the first turns. Losses arise from two main sources: $\mathrm{H}^{-}$ions missing the foil, or not being converted to protons, and protons hitting the walls during the accumulation process. Accsim counts lost particles and takes them off the tracking cycle. A general strategy is to limit the losses to a specific region of the ring (controlled losses). To decrease the beam diffusion, we plan to extract the beam immediately after the injection is completed. To study losses to the level of $10^{-4}$, the number of macro particles in the simulation is chosen between $10^{4}$ and $10^{6}$, with a limit set only by computing time.

\section{MACHINE LATTICE. INJECTION}

The lattice of the accumulator ring, with super symmetry 4 [2], consists of an array of FODO cells with four straights, for injection, collimation, to accommodate the $R F$, and extraction, respectively. The lattice is optimized with $\mathrm{Mad}$ [4], that produces the 51 matrices used in tracking. In the simulation, to account for wall beam losses, a set of collimating apertures were placed at various points around the circumference.

During injection [3], the $\mathrm{H}^{-}$are converted to $\mathrm{H}^{+}$in a $400 \mu \mathrm{g} / \mathrm{cm}^{2}$ carbon stripping foil. A plural tabulated scattering distribution [7] is used to generate scattering angles

\footnotetext{
* Work sponsored by the Division of Material Sciences, U.S. Department of Energy, under contract DE-AC05-960R22464 with Lockheed Martin Energy Research Corp. for Oak Ridge National Laboratory
}

Table 1: Parameters of the injected beam.

\begin{tabular}{|cccc|}
\hline & $\alpha$ & $\beta[\mathrm{m}]$ & $\epsilon$ [mm-mrad] \\
radial: & 0.0014 & 19.140 & 0.35 \\
vertical: & 0. & 4.150 & 0.35 \\
longitudinal: $\Delta \phi=240^{\circ}, \mathrm{dE} / \mathrm{E}=0.001$ \\
\hline
\end{tabular}

in the foil. Nuclear scattering is not currently simulated in Accsim.

The injected number of protons in the accumulator ring is $1.042 \times 10^{14}$, for a $1 \mathrm{MW}$ machine. The injection takes about $1 \mathrm{msec}$, or 1,200 turns This number of tums and the longitudinal phase bite injected, $240^{\circ}$ or $2 / 3$ of the bucket, were chosen to best match the linac beam structure.

At injection, the ring equilibrium orbit is distorted with a radial and a vertical bump. Initially, the beam is directed to the foil in correspondence with the center of the phase space acceptance ellipse of the ring, both radially and vertically. The bumps collapse in time, so that at the end of injection the beam sits on a less bumped radial orbit in the horizontal plane.

The injection line dispersion was matched to the ring dispersion at the foil. Among the other twiss functions, only $\alpha$ was matched, while we tried several radial and vertical $\beta$ values. Typical injection parameters are given in Table 1 .

The distribution of macro particles at injection is taken as random gaussian in the radial and vertical phase space, and flat in the longitudinal phase. The values for the transverse emittance shown in the Table are for $2 \sigma$.

\section{RF CAPTURE. BEAM LOSSES}

Since the beam will be extracted soon after the 1,200 turn injection process is completed, long term beam accumulation and RF capture beyond $1 \mathrm{msec}$ are of no concern. For such a high intensity beam, its size and stability during injection and RF capture are strongly affected by space charge. Tune shifts and tune spread are indicative of transverse space charge effects and should be kept small. A design limit for transverse tune shift is $\Delta \nu=-0.2$. Space charge issues will be discussed in more detail in Sec.4.

Following our experience with AGS operation, and in agreement with previous calculations [5], we also studied the performance of vertically hollow, or "smoke ring" beam, that seems to reduce the tune shift. To obtain a (vertical) smoke ring, during injection we turned off the vertical bump. Beam stacking was studied with two different RF systems [6]: (i) employing the 1.st (or fundamental) har-

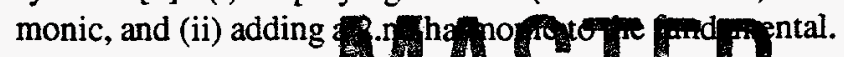


Both $R F$ waveforms are shown in Figure 1.

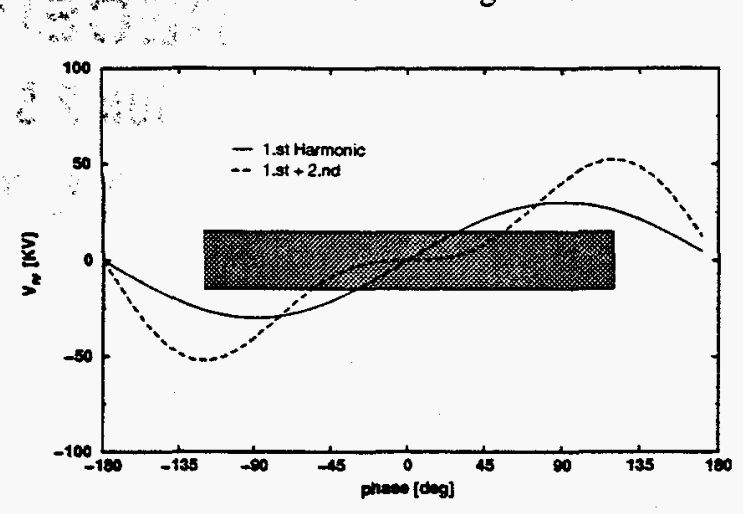

Figure 1: 1.st harmonic (solid line), and 1.st +2 .nd harmonic cavity voltage (dashed line). A $240^{\circ}$ beam bunch is also diagrammatically shown.

Case (i). With the orbit bump, we found a solution with no beam losses in excess of $10^{-4}$ during the 1,200 turns of injection and capture, at a peak accelerating voltage of 40 KV. The resulting bucket area was $6.67 \mathrm{eV}$-sec. Transverse emittance tune shifts in the center of the beam were well contained within the design limits of $\mathbf{- 0 . 2}$.

Case (ii). A good solution, shown in Figure 2, with losses $<10^{-4}$ was also found, at a peak accelerating voltage of $40 \mathrm{KV}(h=1)$ and $20 \mathrm{KV}(h=2)$. Adding a 2.nd harmonic to the RF system improves the longitudinal phase space and the transverse tune shift [6] and creates a more compact beam. The bucket is less than $2 \mathrm{eV}$-sec (plus halo). Figure 3 shows the emittance and tune shifts for this case.
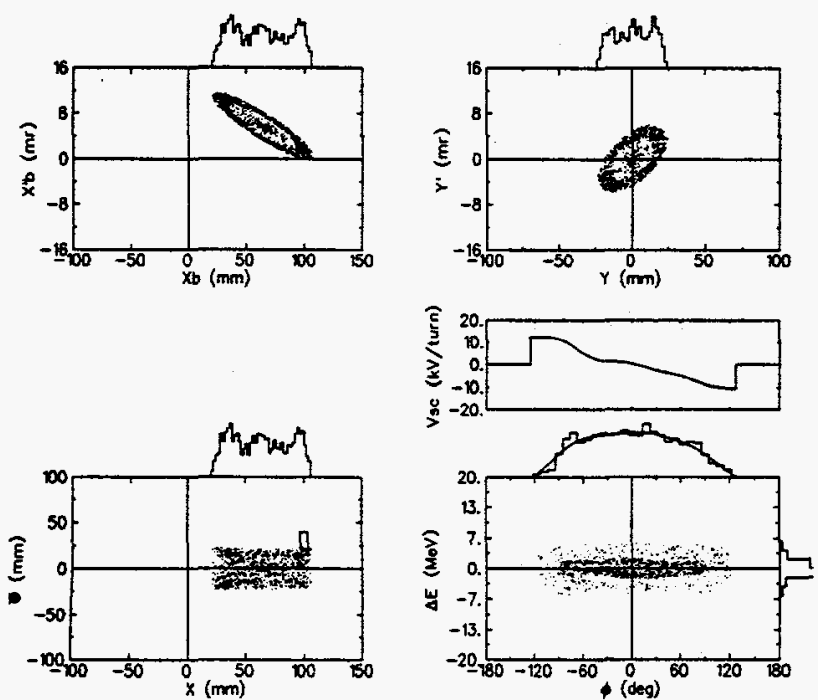

Figure 2: Accsim snapshots at 1,200 turns. 1.st +2 .nd harmonics RF. $1 \mathrm{MW}$ accumulator.

Calculations were repeated for a vertical smoke ring in the conditions of case (ii) above. Losses and foil traversals for the 3 scenarios are are given in Table. 2

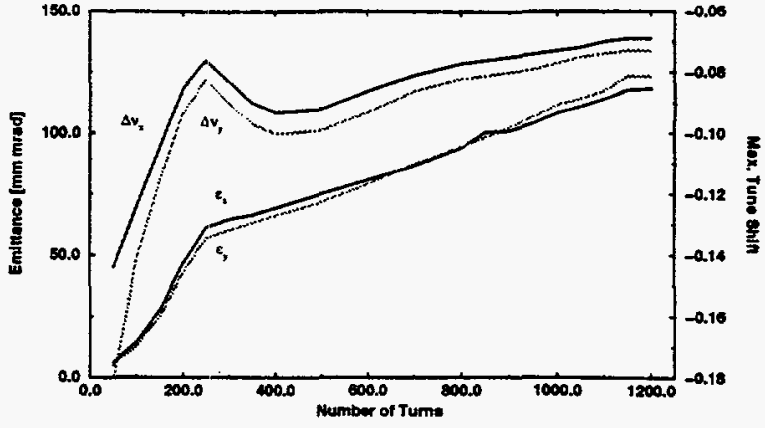

Figure 3: Transverse emittance and tune shift. 1.st $+2 . n d$ harmonics RF. $1 \mathrm{MW}$ accumulator.

Table 2: Losses and foil hits. $1 \mathrm{MW}$ accumulator.

\begin{tabular}{|lccc|}
\hline Macroparticles $=120,000$, & Total charge $=1.042 \times 10^{14}$ \\
lost[\%] & at foil & elsewhere & ave. foil hits \\
1.st harm. RF: & 1.962 & $<8 \times 10^{-5}$ & 3.612 \\
1.st + 2.nd: & 1.867 & $<8 \times 10^{-5}$ & 3.592 \\
smoke ring: & 1.867 & $<8 \times 10^{-5}$ & 3.592 \\
\hline
\end{tabular}

\section{SPACE CHARGE TUNE SPREAD}

The transverse tune shift is calculated in Accsim for each macro particle with a formalism which computes the amplitude dependent tune shift due to the space charge forces of the instantaneous 2-dimensional betatron amplitude distribution, with walls removed. Individual particles are assumed to perform prescribed oscillations within the beam [8].

In the longitudinal dynamics Accsim includes space charge with perfectly conducting smooth walls. The additional space charge voltage induced on the beam is calculated with [10]

$$
V_{s c}=-\frac{Z_{0} c}{4 \pi}\left(1+2 \ln \frac{b}{a}\right) \frac{d \lambda}{d s}
$$

with $Z_{0}$ is the impedance of free space, $d \lambda / d s$ the longitudinal gradient of the particle distribution in the beam, and $b / a$ the ratio between beam and chamber radii $(b / a=3$ in our simulation). At the present, no detailed longitudinal impedance budget is included in Accsim.

A general expression for the (maximum) transverse tune shift induced by space charge in the center of a beam is [9]

$$
\Delta \nu=-r_{0} \frac{\lambda}{\beta^{2} \gamma^{3} \nu} \frac{g}{r}
$$

with $r_{0}$ the classical proton radius, $\lambda$ the longitudinal linear charge density, $\beta$ and $\gamma$ the relativistic velocity and energy, $\nu$ the bare tune, and $g / r$ a form factor that describes the distribution of particles in the beam. Accsim agrees with the above expression when the beam transverse profile has only one peak (pseudo gaussian distribution), but the algorithm that evaluates the tune distribution in the beam (i.e. the tune spread $\Delta \nu$ ), does not provide good accuracy for the general 
case (say, a smoke ring). Work is in progress to calculate space charge forces in a more complete way [11].

In dealing with space charge, the immediate problem is that the on line calculation can be very time consuming and therefore impractical when a very large number of representative particles are used. The evolving charge distribution should be continuously updated to calculate transverse kicks and the tune associated with each particle, in order to evaluate the diffusion of the beam and the growth rate of possible destructive resonances.

Far from walls, the transverse space charge forces on a particle at $P$ can be calculated by an integration on the actual charge distribution (it is $\xi=x$, or $y$ )

$$
F_{\xi}(P)=\frac{\mu_{0} e^{2} c^{2} n}{4 \pi \gamma^{2}} \int \frac{\rho(Q)}{r^{3}} \xi d Q,
$$

with $n e$ the charge contained in a beam volume $V$. Taking into account the relativistic longitudinal compression of the field, the preceding integral describes the interaction between filaments of current for not too high energies (our case) and point interaction for extreme energies.

The space charge force produces a tune shift $\Delta \nu$ at each macro particle location. To calculate the tune at $P$, consider the betatron equation in $r$ (either $x$ or $y$ ):

$$
\frac{d^{2} \xi}{d z^{2}}+K_{\xi} \xi=f(\xi, z)=\frac{F_{\xi}}{m_{0} \gamma \beta^{2} c^{2}} .
$$

The space charge force is zero in the center of the beam and is anti symmetric in $\xi$, because the net force is repulsive on both sides. The tune shift near the center will be represented by the radial derivative of $f$. We deal in general with a non linear equation that generates oscillations with a variety of frequencies. The r.h.s. contains both $x$ and $y$ and generates also coupling beat frequencies. With numerical integration followed by a FFT analysis we could calculate the tune distribution in the beam and the coupling frequencies. The calculated radial component of the space charge force for a pseudo gaussian and for a smoke ring is shown in Figure 4. The figures show how the force derivative in the center can be much smaller for a smoke ring. Results of the FFT analysis shows that the tune shift and tune spread are smaller for a smoke ring, also when averaged over all particles in the beam. The FFT shows also the coupling beats.

An example of "necktie" diagrams, taken every 135 turns during beam stacking, for a $2 \mathrm{MW}$ machine, are in Figure 5 . The figure clearly shows that the necktie area increases very fast when the total beam charge is approaching its upper limit. .

\section{REFERENCES}

[1] F.WJones: "User's Guide to Accsim". TRIUMF Design Note TRI-DN-90-17, June 1990 (and later additions).

[2] Y.Y.Lee: Proceedings of this Conference.

[3] L.Blumberg: Proceedings of this Conference.
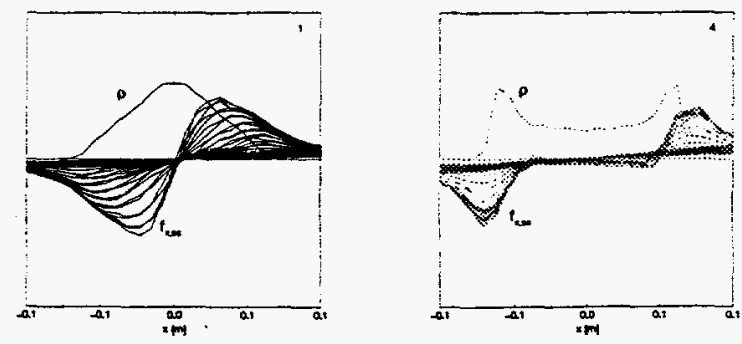

Figure 4: Pseudo Gaussian. Density and Space charge force vs. $x$ for various $y$.

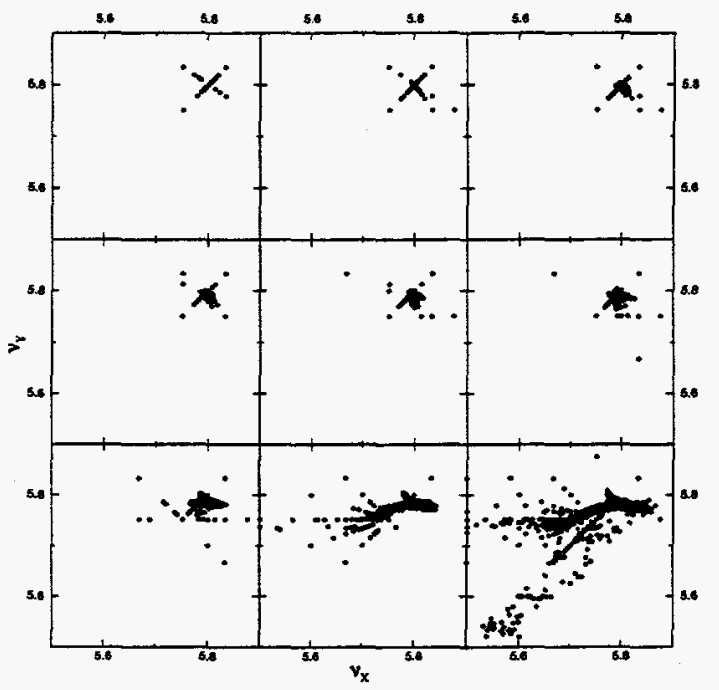

Figure 5: Distribution of peaks in the tune chart. 256 particles. Pseudo Gaussian population.

[4] F.Ch. Iselin and J. Niederer. "The Mad Program. User's Reference Manual". CERN/LEP-TH/88, July 13, 1988 (and later additions).

[5] L.N. Blumberg, A.U. Luccio, A. VanSteenbergen, F.W. Jones. BNL-62391, UC-414, AGS/AD/96-2, Upton, 1995.

[6] M. Blaskiewicz, J.M. Brennan and Y.Y.Lee. Proceedings this Conference

[7] E. Keil, E. Zeitler and W. Zinn. Z. Naturf. 15a,1031,1960.

[8] H. Schönauer. TRIUMF Design Note TRI-DN-89-K50, August 1989.

[9] A.W. Chao: 'Physics of Collective Beam Instabilities in High Energy Accelerators', Wiley, New York 1993

[10] A. Hoffman, CERN 77-13,1977.

[11] A.U. Luccio, BNL/NSNS Tech. Note No.023, Upton, 1997. 


\section{Disclaimer}

This report was prepared as an account of work sponsored by an agency of the United States Government. Neither the United States Government nor any agency thereof, nor any of their employees, makes any warranty, express or implied, or assumes any legal liability or responsibility for the accuracy, completeness, or usefulness of any information, apparatus, product, or process disclosed, or represents that its use would not infringe privately owned rights. Reference herein to any specific commercial product, process, or service by trade name, trademark, manufacturer, or otherwise does not necessarily constitute or imply its endorsement, recommendation, or favoring by the United States Government or any agency thereof. The views and opinions of authors expressed herein do not necessarily state or reflect those of the United States Government or any agency thereof. 


\section{DISCLAIMER}

Portions of this document may be illegible in electronic image products. Images are produced from the best available original document. 